\title{
On the 100 Gbps DWDM with Optical Carrier Suppression Modulation
}

https://doi.org/10.1515/comp-2020-0104

Received Mar 27, 2020; accepted Sep 04, 2020

\begin{abstract}
This paper investigates the transmission performance of 16-channel DWDM (Dense Wavelength Division Multiplexing) system with two complex Optical Differential Quadrature Phase Shift Keying modulator configurations using 2 LiNb MZM (Mach-Zehnder Modulator) and 3 LiNb MZM. The link performance is evaluated for $100 \mathrm{Gbps}$ data rate per channel in a total $750 \mathrm{~km}$ single mode fiber link. The perfomance is analyzed in terms of forward power, reflected power and bit-error rate of the received signal. From the simulation results we prove, that the link performance can be improved by adopting the high efficiency optical modulation.
\end{abstract}

Keywords: CSRZ-DQPSK, DWDM, nonlinear effects

\section{Introduction}

Recent research in the field of fiber optical communication focuses on increasing the link capacity in order to meet the demand of current broadband applications. Over the years multiple approaches of costeffective broadband transmission systems have been studied. One way to increase the link capacity is to implement less-complex broadband optical transmitters. Optical Differential Quadrature Phase Shift Keying (DQPSK) is the most reliable of optical modulation techniques. This modulation technique is known for its high spectral efficiency and the high data rate. Optical DQPSK modulation technique can be implemented

\footnotetext{
*Corresponding Author: Tomáš Huszanık: Department of Electronics and Multimedia Communications, Faculty of Electrical Engineering and Informatics, Technical University of Košice, Košice, Slovakia; Email: tomas.huszanik@tuke.sk

Ján Turán: Department of Electronics and Multimedia Communications, Faculty of Electrical Engineering and Informatics, Technical University of Košice, Košice, Slovakia; Email: jan.turan@tuke.sk Luboš Ovsenık: Department of Electronics and Multimedia Communications, Faculty of Electrical Engineering and Informatics, Technical University of Košice, Košice, Slovakia;

Email: lubos.ovsenik@tuke.sk
}

using 2 Mach-Zehnder optical modulators (MZM). The main limitation of the fiber optical communication is the signal degradation due to fiber nonlinear effects and chromatic dispersion. This limitation is even more problematic in high capacity DWDM (Dense Wavelength Division Multiplexing) systems as was investigated in [1].

In order to mitigate fiber nonlinear effects, an optical modulation with suppressed carrier can be applied. Such optical modulation technique is called Carrier Suppressed Return to Zero Differential Quadrature Phase Shift Keying (CSRZ-DQPSK) [1]. It is implemented using 3 MZM based optical transmitter. CSRZ based optical modulation can provide better tolerance to fiber nonlinear effects and chromatic dispersion.

The aim of this paper is to provide a comparative analysis of two optical modulation techniques, DQPSK and CSRZDQPSK, in a DWDM system with capacity of $16 \times 100 \mathrm{Gbps}$. The two modulation techniques are analysed in term of their input power, reflected power and received bit-error rate (BER).

\section{Carrier suppressed optical modulator structure}

The conventional optical DQPSK modulator structure, based on two arms LiNb MZM driven by in-phase and quadrature-phase signal components, is shown on Figure 1.

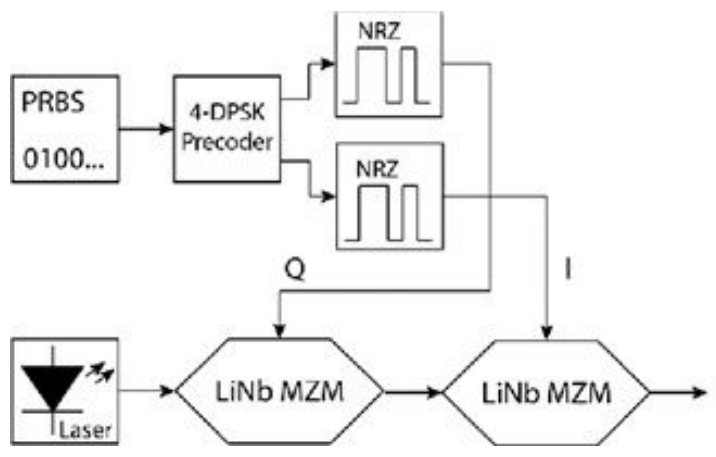

Figure 1: Two LiNb MZM DQPSK modulator structure. 
The modulator structure shown in Figure 1 is commercially available in a $\mathrm{LiNbO}_{3}$ integrated form [4]. There is a $\pi / 2$ phase shift between the I and Q signal component. Data are generated by PRBS (Pseudo Random Bit Sequence), with a data rate of $100 \mathrm{Gbps}$ which are then precoded by a 4-DPSK precoder and finally shaped by electrical NRZ (Non Return to Zero) driver. The 4-DPSK precoder is used to prevent recursive decoding on the receiver side. The precoder performs the following mathematical operations:

$$
\begin{aligned}
& I_{i}=\overline{\left(Q_{i-1} \oplus I_{i-1}\right)\left(U_{i} \oplus I_{i-1}\right)}+\left(Q_{i-1} \oplus I_{i-1}\right) \overline{\left(V_{i} \oplus I_{i-1}\right)} \\
& Q_{i}=\overline{\left(Q_{i-1} \oplus I_{i-1}\right)\left(V_{i} \oplus I_{i-1}\right)}+\left(Q_{i-1} \oplus I_{i-1}\right) \overline{\left(U_{i} \oplus I_{i-1}\right)}
\end{aligned}
$$

In the above formulas, $\oplus$ donates the logical operation of XOR (Exclusive OR). $U_{i}$ and $V i$ are the input signals generated by PRBS. $I_{i}$ and $Q_{i}$ are the DQPSK coded output signals. Then, the logic operation $\overline{I_{i-1}}, \overline{Q_{i-1}}$ corresponds to the logic state 00 and the $180^{\circ}$ phase difference. The logic operation $Q_{i-1}, I_{i-1}$ corresponds to logic state 01 and $90^{\circ}$ phase difference. $270^{\circ}$ phase difference matches logic state 10 and $Q_{i-1}$, $I_{i-1}$ logic operation. $I_{i-1}, Q_{i-1}$ corresponds to logic state 11 and $0^{\circ}$ phase difference $[1-3]$.

The DQPSK modulator conveys information by establishing a phase of one symbol relative to the previous symbol while QPSK conveys the absolute phase of each symbol. This means, that standard DQPSK phase options are $0^{\circ}$, $90^{\circ},-90^{\circ}$ and $180^{\circ}$ or $-180^{\circ}$ while phase options of QPSK are $45^{\circ}, 135^{\circ}, 225^{\circ}$ and $315^{\circ}$.

The induced phase difference between two arms of the modulator can be expressed by equations [3-5]:

$$
\Delta \varphi_{I}(t)=\frac{u_{I}(t)}{V_{\pi}} \pi, \Delta \varphi_{Q}(t)=\frac{u_{Q}(t)}{V_{\pi}} \pi
$$

where $\Delta \varphi_{I}(t)$ and $\Delta \varphi_{Q}(t)$ are phase differences of $I$ and $Q$ signal components, $u_{I}(t)$ and $u_{Q}(t)$ are the applied voltage and $V_{\pi}$ is the half-wave voltage. The transfer function of the IQ modulator is [4]:

$$
\frac{E_{\text {out }}(t)}{E_{\text {in }}(t)}=\frac{1}{2} \cos \left(\frac{\Delta \varphi_{I}(t)}{2}\right)+j \frac{1}{2} \cos \left(\frac{\Delta \varphi_{Q}(t)}{2}\right)
$$

Using these two equations it is possible to calculate amplitude modulation $A_{I Q M}(t)$ (3) and phase modulation $\varphi_{I Q M}(t)(4)$ of $I Q$ modulator [4].

$$
\begin{gathered}
A_{I Q M}(t)=\left|\frac{E_{\text {out }}(t)}{E_{\text {in }}(t)}\right|=\frac{1}{2} \sqrt{\cos ^{2}\left(\frac{u_{I}(t)}{V_{\pi}} \pi\right)+\cos ^{2}\left(\frac{u_{Q}(t)}{V_{\pi}} \pi\right)} \\
\varphi_{I Q M}(t)=\arg \left[\cos \left(\frac{u_{I}(t)}{V_{\pi}} \pi\right), \cos \left(\frac{u_{Q}(t)}{V_{\pi}} \pi\right)\right]
\end{gathered}
$$

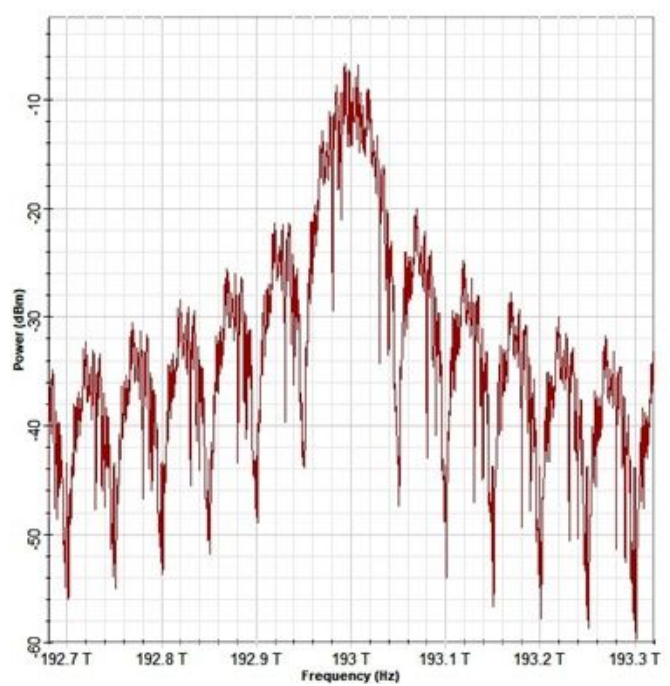

Figure 2: Optical spectra of 2 MZMs based DQPSK modulated signal.

The signal spectra of a 2 MZM based optical DQPSK modulator emitting on $193 \mathrm{THz}$ is shown in Figure 2.

Fiber non-linear effects, especially SMP and XPM, are energy sensitive. The negative contribution of these effects is well known and intensively studied. Another problem is the instability of modulation, which affects the performance of optical communication systems in which losses are regularly compensated by an optical amplifier (such as DWDM systems). The results of the simulation showed that the main limiting factor is the use of Non-Return to Zero (NRZ) format for data transmission [710].

A possible way to mitigate nonlinear effects and to achieve increased DWDM performance is to use carriersuppressed optical modulation. CSRZ-DQPSK (Carrier Suppressed Return to Zero DQPSK) is one of the most promising optical modulation schemes for long distance and high data rate optical systems, providing increased tolerance to nonlinear effects and chromatic dispersion.

The CSRZ-DQPSK optical modulator may consist of 3 LiNb MZM as shown in Figure 3.

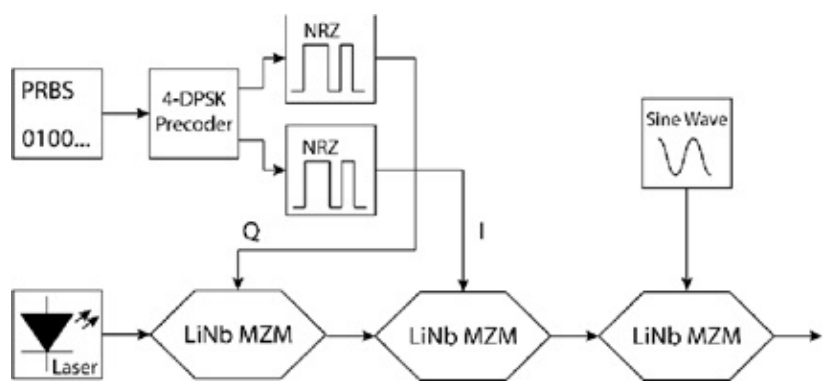

Figure 3: 3 MZMs DQPSK modulator structure (CSRZ-DQPSK). 
The first MZM is used to generate a phase shift of 0 or $\pi$ of the phase signal component. The second MZM generates a quadrature phase shift of 0 or $\pi / 2$ modulating the quadrature phase signal component. Possible phase differences at the output of the second MZM are $0, \pi / 2,3 \pi / 2$ and $\pi$. A third MZM is used to generate the carrier-suppressed signal. It is used as a pulse carver. Pulse cutting is performed by driving the MZM sine wave RF signal at a frequency of one quarter of the bit rate. The peak-to-peak amplitude is $\mathrm{V} \pi$. The signal spectra of the CSRZ-DQPSK optical modulator based on 3 MZMs emitting $193 \mathrm{THz}$ are shown in Figure 4 $[11,12]$.

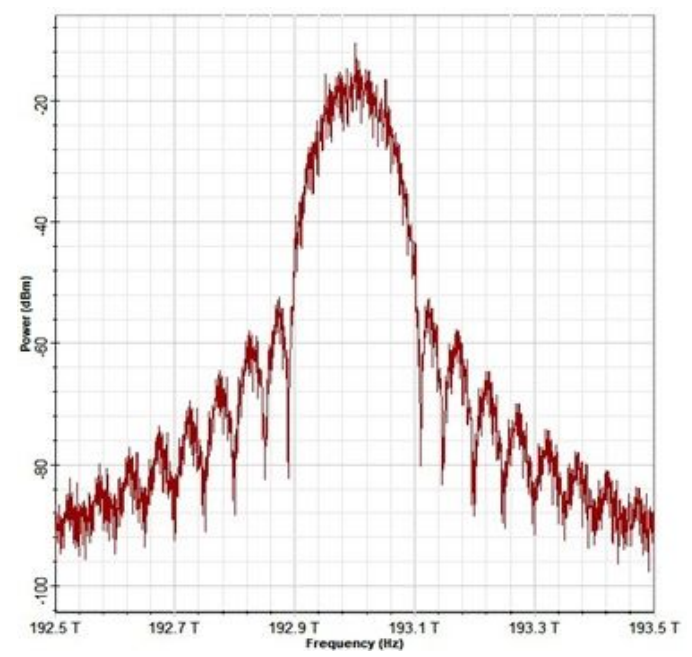

Figure 4: Optical spectra of CSRZ-DQPSK modulated signal.

\section{Proposed DWDM simulation model}

The 16-channel DWDM simulation model is designed in the OptiSystem $^{\mathrm{TM}}$ simulation software.

The block diagram of the proposed simulation model is shown in Figure 5. The simulation model consists of three subsections: a transmitter, an optical distribution and a receiving section. General simulation features are: bit rate of $100 \mathrm{Gbps}$, time window is $2.56 \mathrm{e}-09 \mathrm{~s}$, sample rate of 320 $\mathrm{GHz}$ a sequence length of $256 \mathrm{bits}$. The number of wavelength channels is 16 . The channel wavelengths are set according to ITU-T G.694.1. The simulation is performed in the optical band C (1530-1565 nm). The channels are then multiplexed by a 16-channel waveguide grid multiplexer (AWG) with $5.5 \mathrm{~dB}$ attenuation (standard value of 16-channel AWG miltiplex).

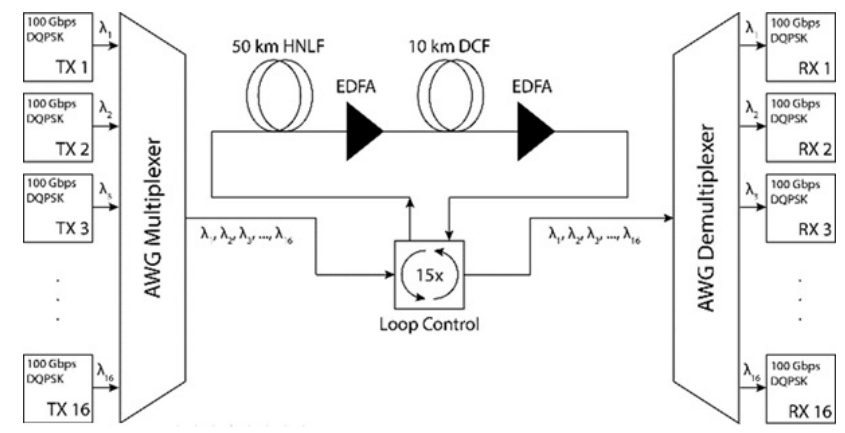

Figure 5: 16-channel 100 Gbps DQPSK DWDM system.

The optical distribution path consists of $50 \mathrm{~km}$ of highly nonlinear single mode optical fiber (HNLF), two erbiumdoped fiber amplifiers (EDFA) and $10 \mathrm{~km}$ of dispersion compensating fiber (DCF). The optical distribution path is located in the loop component. The number of loops is 15 (750 km). An advanced SMF simulation model is used. This means that both linear and non-linear effects are taken into account. The attenuation is $0.25 \mathrm{~dB} / \mathrm{km}$, the chromatic dispersion is $16 \mathrm{ps} / \mathrm{km}-\mathrm{nm}^{2}$, the non-linear refractive index is $\mathrm{n}_{2}=2.6 \mathrm{e}-20 \mathrm{~m}^{2} / \mathrm{W}$ and the effective fiber cross-sectional area is $\mathrm{A}_{\text {eff }}=80 \mu \mathrm{m}^{2}$. The chromatic dispersion is fully compensated in DCF with the dispersion set at $-80 \mathrm{ps} / \mathrm{km}-\mathrm{nm}^{2}$. Channels are demultiplexed with a AWG demultiplexer with $5.5 \mathrm{~dB}$ insertion loss.

Received signal is demodulated in a $90^{\circ}$ optical hybrid shown in Figure 6. Received signal is analyzed in electrical domain and from the received samples, the BER and Qfactor is calculated [6]:

$$
Q=\frac{I_{1}-I_{0}}{\sigma_{1}+\sigma_{0}} .
$$

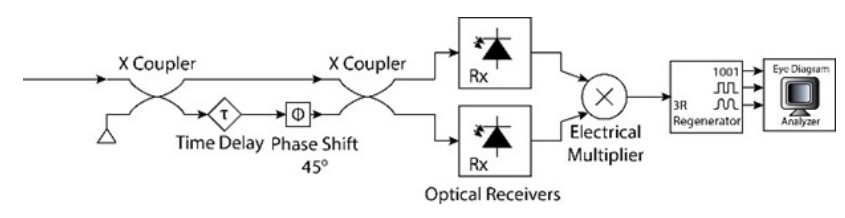

Figure 6: Noncoherent DQPSK receiver.

In the equation (7), $I_{1}$ is the mean value and $\sigma_{1}$ is the deviation of the pulse $1, I_{0}$ is the mean value and $\sigma_{0}$ is the deviation of pulse 0 . The bit error rate (BER) is calculated as follows:

$$
B E R=\frac{1}{2} \operatorname{erfc}\left(\frac{Q}{\sqrt{2}}\right) \approx \frac{1}{\sqrt{2 \pi} Q} \exp \left(-\frac{Q^{2}}{2}\right) .
$$

The threshold for the BER value is 1.0e-10 with Q-factor 6.3613. The performance of proposed DWDM system is not acceptable for BER higher than 1.0e-10. 
Simulation results are presented for received channel no. 5 (193.4 THz).

\section{Simulation results}

The performance of NRZ-DQPSK and CSRZ-DQPSK modulated 16-channel DWDM system with the data rate of 100 Gbps per channel was firstly analyzed over 15 optical segments. Each segment includes $50 \mathrm{~km}$ of HNFL, $10 \mathrm{~km}$ of DCF and two EDFAs.

Figure 7 and Figure 8 show comparison between forward and reflected power of the NRZ-DQPSK and

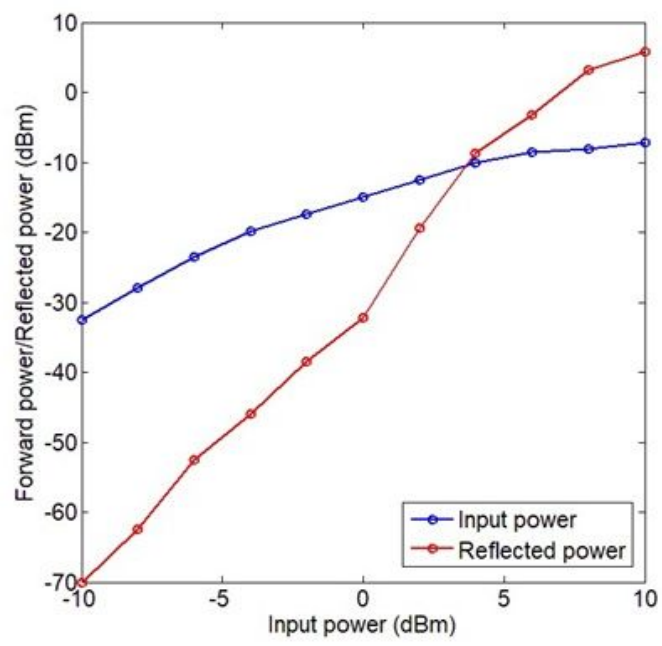

Figure 7: Forward and Reflected Power for DQPSK modulation.
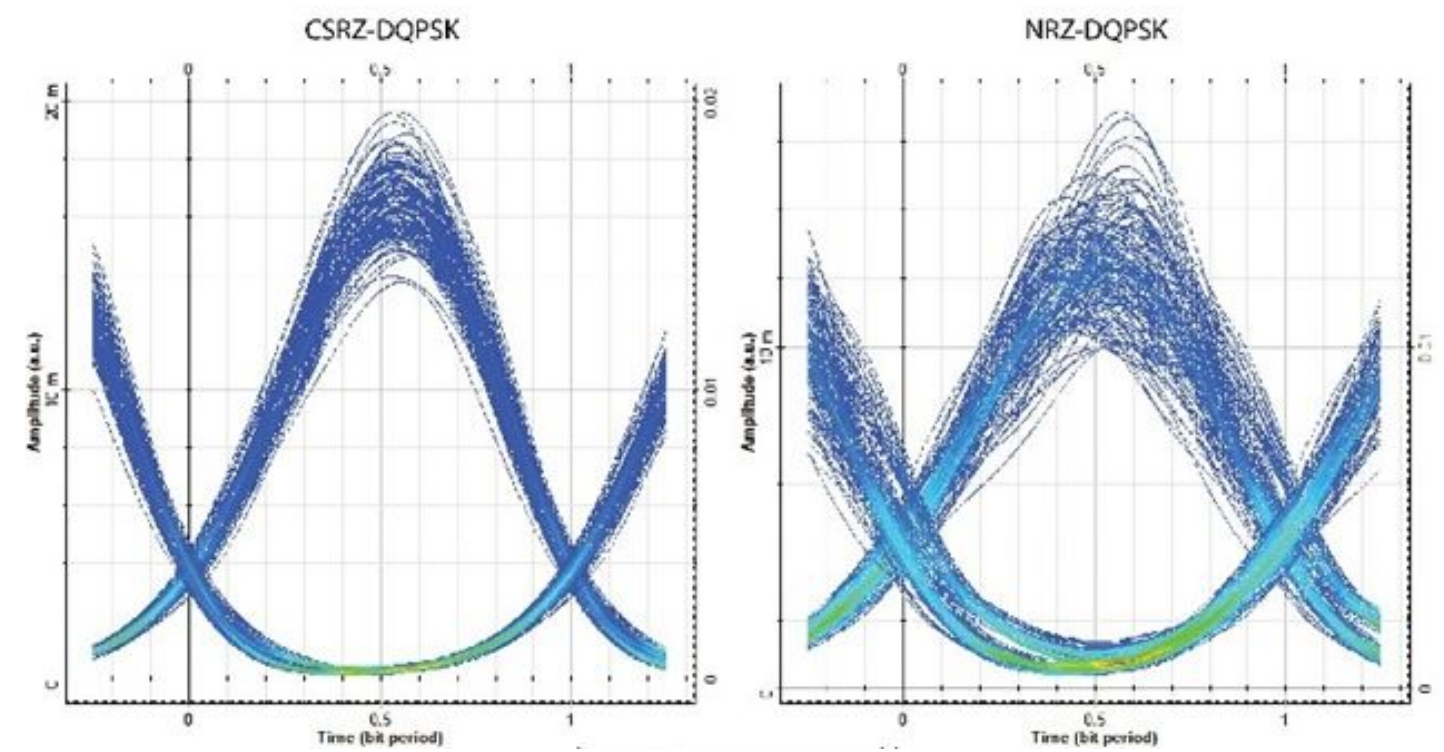

CSRZDQPSK transmitter. The most important parameters of the eye diagram are the eye opening and the thickness of the lines. CSRZ-DQPSK modulated DWDM system shows better eye opening which indicates lower bit error rate (BER) and higher Q-factor. NRZ-DQPSK modulated DWDM system shows less opened eye diagram. Thicker the lines of the eye diagram, higher the bit rate. The thickness of the lines also indicates the OSNR (Optical Signal-to-Noise Ratio). In term of these results, the nonlinear effects can be mitigated by deployment of carrier suppressed optical modulation.

Figure 9 shows the eye diagrams of received signals after 5 loops $(250 \mathrm{~km})$. Figure 10 shows eye diagrams of the

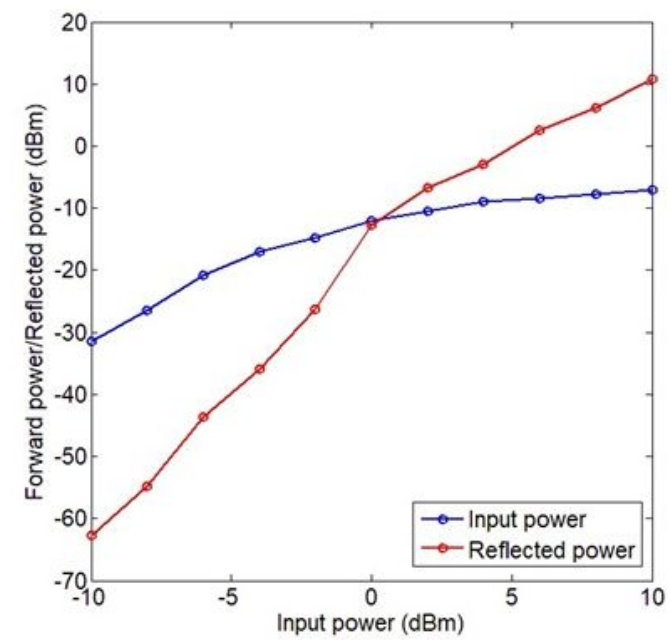

Figure 8: Forward and Reflected Power for CSRZ-DQPSK modulation.

Figure 9: Eye diagrams of received signals after $250 \mathrm{~km}$. 

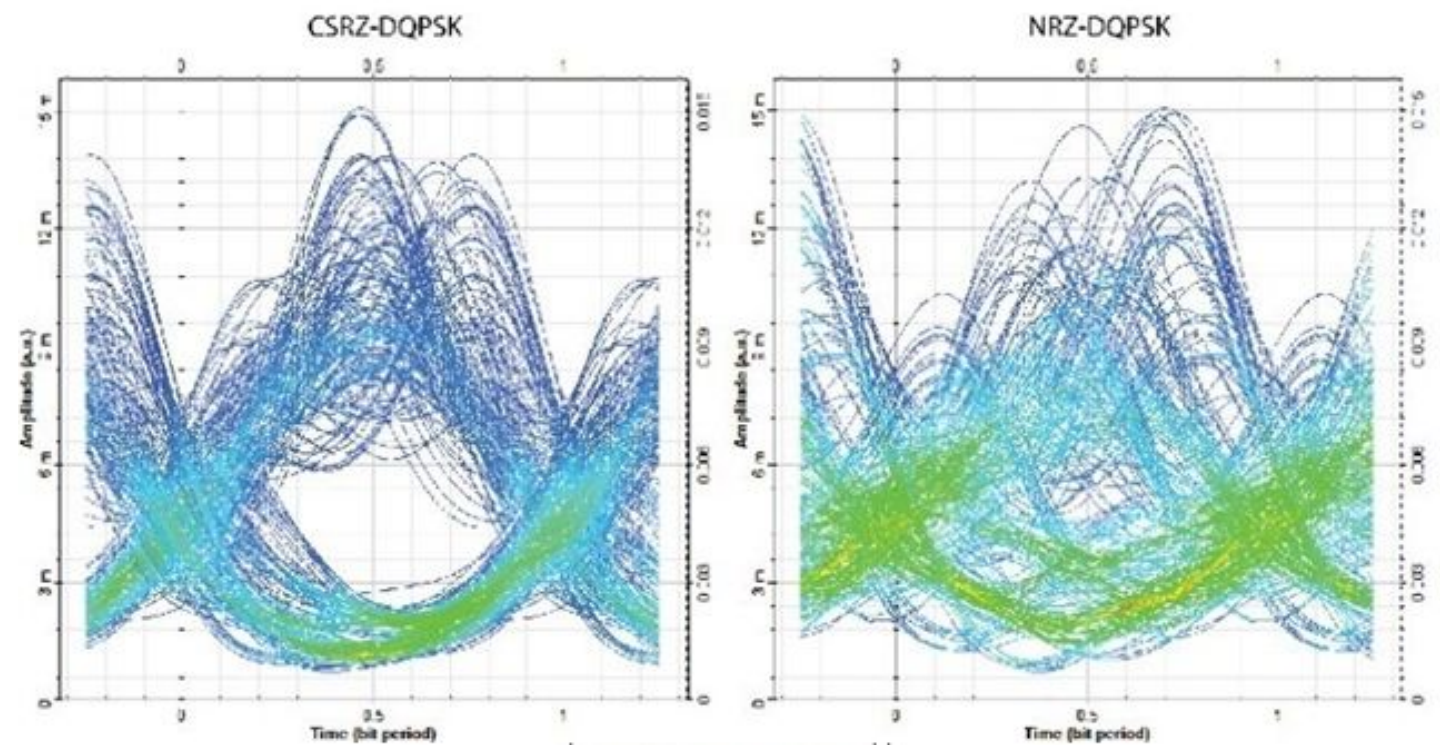

Figure 10: Eye diagrams of received signals after $550 \mathrm{~km}$.

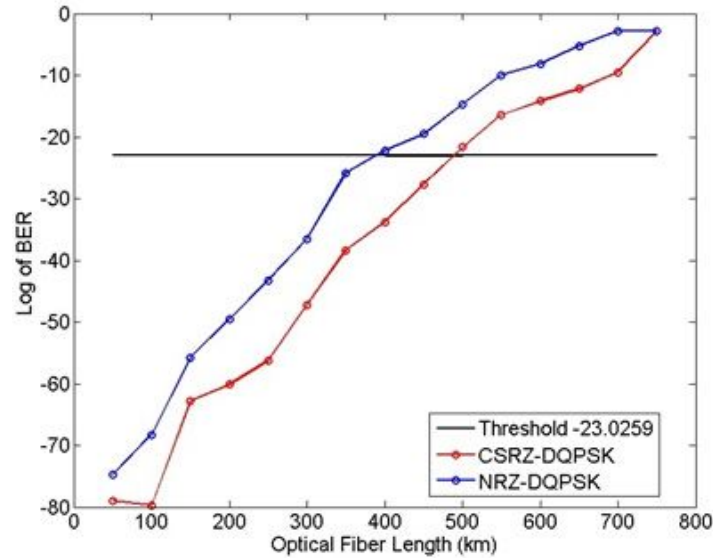

Figure 11: Optical spectra of DQPSK modulated signal.

signals of the same DWDM system received after $550 \mathrm{~km}$ (11 loops).

The dependence between the length of the fiber optical transmission path and the modulation method used in DWDM is shown in Figure 9.

In the next experiment, the effect of increased CW laser launch power on the creation of fiber nonlinear effects is analyzed. The transmission distance is set to $350 \mathrm{~km} \mathrm{(7}$ loops). The CW laser launch power is linearly swept from 0 $\mathrm{dBm}$ to $10 \mathrm{dBm}$. Again, the channel 5 is analyzed and BER is calculated.

Based on the obtained results, the performance of 16channel DQPSK modulated DWDM system can be improved using optical carrier suppression method. Using the three MZMs DQPSK modulator structure, also known as CSRZ-

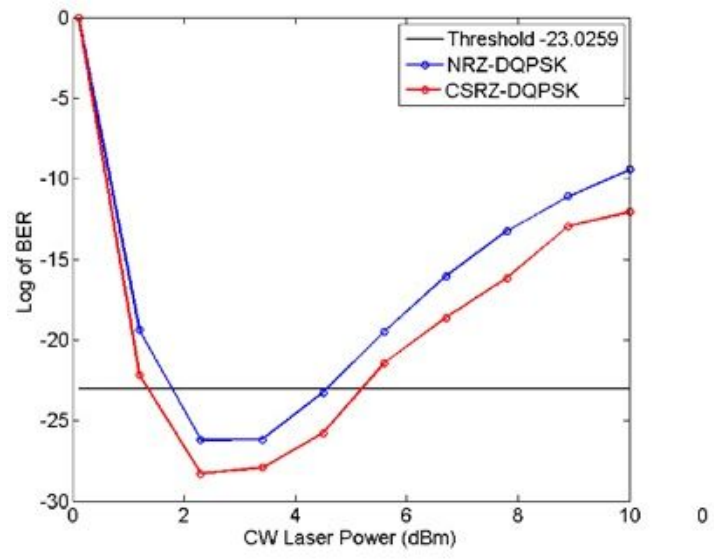

Figure 12: Optical spectra of DQPSK modulated signal.

DQPSK optical modulator, the negative contribution of fiber nonlinear effects can be mitigated. The maximum transmission distance for CSRZ-DQPSK modulated DWDM is 500 $\mathrm{km}$. The BER is 3.96e-010. The BER of NRZ-DQPSK modulated DWDM on the same distance is $4.25 \mathrm{e}-007$ which is inefficient. The launch power is also very important parameter due to the power dependence of SPM and XPM. CSRZ-DQPSK modulated DWMD system is more resistant to nonlinear effects due to the increased launch level. It means that we can manage the power in such DWDM more effectively. It also means that using the same launch level, CSRZ-DQPSK modulated DWDM system has the lower signal attenuation and thus transmission on the greater distance can be achieved. 


\section{Conclusion}

Optical differential quadrature phase shift keying is the most efficient optical modulation technique for transmitting high data rates. In this paper we compared the performance of 100 Gbps 16 channel DWDM system with NRZ DQPSK and CSRZ-DQPSK optical modulation. It is observed that CSRZ-DQPSK result in higher tolerance to fiber nonlinear effects. Thus, higher resistance to nonlinear effects and higher performance can be achieved through the carrier suppressed DQSPK.

Acknowledgement: This work was supported by following research grant: APVV-17-0208 - Resilient mobile networks for content delivery.

\section{References}

[1] T. Huszaník, J. Turán, L'. Ovseník, “Comparative analysis of optical IQ modulation in four-channel DWDM system in the presence of fiber nonlinearities," Proceedings of the 2018 19th International Carpathian Control Conference, ICCC 2018, p. 468-473. DOI:10.1109/CarpathianCC.2018.8399675.

[2] J. M. Simmons, "Optical Network Design and Planning," 2014 , 529 p. ISSN: 1935-3847, DOI: 10.1007/978-3-319-05227-4.

[3] W. Ji and J. Chang, "Design of WDM-RoF-PON for wireless and wire-line access with source-free ONUs," in IEEE/OSA Journal of Optical Communications and Networking, vol. 5, no. 2, pp. 127-133, February 2013. DOI: 10.1364/JOCN.5.000127.

[4] M. Seimetz, "High-Order Modulation for Optical Fiber. Transmission,” Springer-Verlang Berlin Heidelberg, 2009,. ISBN 978-354093770-8.
[5] J. Tóth, L. Ovseník, J. Turán, L. Michaeli, M. Márton, "Classification prediction analysis of RSSI parameter in hard switching process for FSO/RF systems", Measurement, vol. 116, pp. 602-610, 2018, 10.1016/j.measurement.2017.11.044

[6] V. Bobrovs, A. Udalcovs, I. Trifonovs. "Evaluation of Nonlinear Effect Impact on Optical Signal Transmission over Combined WDM System, “ Progress in Electromagnetic Research Symposium (PIERS 2013), Taiwan, Taipei, 25-28 March, 2013. Taipei: The Electromagnetics Academy, pp.303-307, 2013. ISBN 978-1934142-24-0. ISSN 1559-9450.

[7] P. Ivaniga, T. Ivaniga, J. Turán, L.. Ovseník, M. Márton, D. Solus, J. Oravec, T. Huszaník. "The Influence of FWM with AWG Multiplexor in DWDM System,” Przeglad Elektrotechniczny, vol. 2018, no. 4, pp. 113-117, 2018. DOI:10.15199/48.2018.04.28.

[8] W. Steingartner, M. A. M. Eldojali, D. Radakovic, J. Dostal, "Software support for course in semantics of programming languages," Paper presented at the 2017 IEEE 14th International Scientific Conference on Informatics, INFORMATICS 2017 - Proceedings, , 2018 January, p. 359-364. DOI:10.1109/INFORMATICS.2017.8327275.

[9] J. Kahn, K. P. Ho. "Spectral Efficiency Limits and Modulation/Detection Techniques for DWDM Systems," IEEE. Journal of Selected Topics in Quantum Electronics, vol. 10, no. 2, p. 259-272 (2004). ISSN: 1558-4542. DOI: 10.1109/JSTQE.2004.826575.

[10] R. Maharjan, I. Lavrinovica, A. Supe, and J. Porins, "Minimization of FWM effect in nonlinear optical fiber using variable channel spacing technique," Advanced Wireless Optical Communication RTUWO 2016, vol. 3, no. 3, pp. 1-4, 2016, DOI: 10.1109/RTUWO.2016.7821844.

[11] A. Kaur, S. Dewra. "Comparative Analysis of Different Modulation Techniques in Coherent Optical Communication System," International Journal of Innovative Research in Computer and Communication Engineering, vol. 3, issue 8, p. 7193 - 7200, August 2015. ISSN: 2320-9801.

[12] O. Kovac, P. Lukacs, I. Gladisova, "Textures classification based on DWT," Paper presented at the 2018 28th International Conference Radioelektronika, RADIOELEKTRONIKA 2018, p. 1-5. DOI:10.1109/RADIOELEK.2018.8376379. 